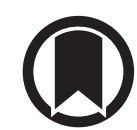

CrossMark

\title{
Tuberculosis after a borderline QuantiFERON result during screening before infliximab
}

\author{
To the Editor:
}

Patients who are eligible for treatment with immunosuppressive drugs such as antagonists of tumour necrosis factor (TNF)- $\alpha$ must be screened for latent tuberculosis infection (LTBI), as stated in the recent European standards for tuberculosis (TB) care [1]. Although there are controversies regarding optimal screening [2], interferon- $\gamma$ release assays such as QuantiFERON-TB Gold (QFT) (Qiagen, Hilden, Germany) are nowadays frequently used. The formal cut-off for a positive QFT is $\geqslant 0.35 \mathrm{IU} \cdot \mathrm{mL}^{-1}$ interferon- $\gamma$. However, a recent study published in the European Respiratory Journal found that a significant proportion of results just below this cut-off, so called borderline results, represented true infection with Mycobacterium tuberculosis [3]. We present a patient who clearly illustrates the clinical significance of borderline QFT results in patients screened before immunosuppression.

December 2017, a 33-year-old woman who was 33 weeks pregnant presented to Leiden University Medical Center (Leiden, the Netherlands) with headache, spiking fever and night sweats starting 3 weeks previously. She was born in Morocco and had moved to the Netherlands in 2003. She had ulcerative colitis for which she was treated with low-dose prednisone, mesalazine and infliximab once per 6 weeks, the latter since June 2017. Her temperature was $39.0^{\circ} \mathrm{C}$, but physical examination revealed no other abnormalities, in particular no neurological signs. Laboratory data showed a C-reactive protein concentration of $56 \mathrm{mg} \cdot \mathrm{L}^{-1}$ (normal value $<10 \mathrm{mg} \cdot \mathrm{L}^{-1}$ ) and a normal white-cell count. A chest radiograph showed hilar lymphadenopathy and a diffuse nodular pattern, suspicious of miliary TB. Computed tomography imaging of the brain showed no signs of cerebral TB and cerebrospinal fluid was without abnormalities. Standard quadruple TB therapy was immediately started. The QFT result was positive (TB1 $5.37 \mathrm{IU} \cdot \mathrm{mL}^{-1}$, TB2 $\left.5.55 \mathrm{IU} \cdot \mathrm{mL}^{-1}\right)$. The clinical course is shown in figure 1. Auramine staining and PCR for M. tuberculosis on gastric lavage fluid and sputum were positive. Mycobacteria growth indicator tube cultures of gastric lavage fluid and sputum cultures became positive for M. tuberculosis after 15 days. Susceptibility testing later showed full susceptibility.

The pre-anti-TNF screening was reviewed. 5 months prior to admission, she had been screened for LTBI in a local hospital when she was not yet using any immunosuppressive therapy. The patient was bacille Calmette-Guérin (BCG)-vaccinated and had reported contact with a relative with active TB 20 years earlier. She had visited Morocco several times since 2003. Her tuberculin skin test (TST) result was $10 \mathrm{~mm}$ induration, which appeared to be overlooked by the attending physician, and QFT test result was negative (TB1 $0.11 \mathrm{IU} \cdot \mathrm{mL}^{-1}$, TB2 $0.22 \mathrm{IU} \cdot \mathrm{mL}^{-1}$ ). Because of the pregnancy, no chest radiography had been performed at that time. Thus no preventive therapy was started. The original colon biopsies were reviewed, but no granulomas were found.

During TB treatment, mesalazine and prednisone were continued but infliximab therapy was withheld. The patient had a favourable clinical response and 20 days later, gave birth to a healthy daughter without signs of congenital TB and negative histology of the placenta. The newborn was given isoniazid preventive therapy, which was discontinued after the tuberculin skin test was negative 3 months later. Variable-number tandem repeat (VNTR) typing of the patient's $M$. tuberculosis isolate was performed by the National Institute for Public Health and the Environment (Bilthoven, the Netherlands), showing a unique VNTR number (9007047), which strongly argues in favour of exposure abroad.

@ERSpublications

Development of tuberculosis after a borderline QuantiFERON result during screening before TNF- $\alpha$ antagonist therapy http://ow.ly/zW1u30kw2Qv

Cite this article as: Uzorka JW, Delfos NM, Witte AMC, et al. Tuberculosis after a borderline QuantiFERON result during screening before infliximab. Eur Respir J 2018; 52: 1800913 [https://doi.org/ 10.1183/13993003.00913-2018]. 


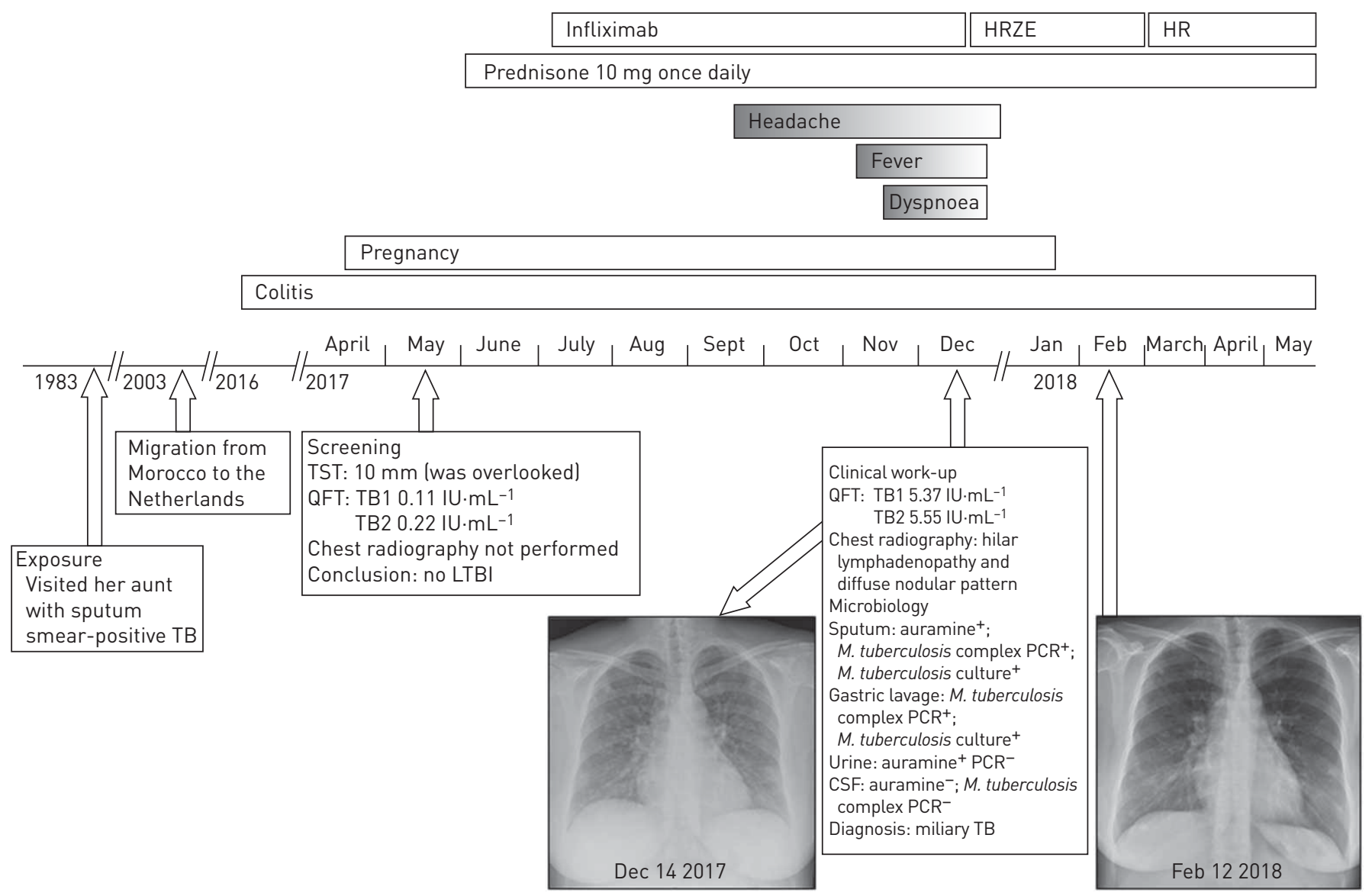

FIGURE 1 Timeline of the clinical course. H: isoniazid; R: rifampicin; Z: pyrazinamide; E: ethambutol; TB: tuberculosis; TST: tuberculin skin test; QFT: QuantiFERON-TB Gold Plus; LTBI: latent tuberculosis infection; M. tuberculosis: Mycobacterium tuberculosis; CSF: cerebrospinal fluid.

This patient, who developed miliary TB during pregnancy and after starting infliximab, preceded by a negative QFT result in the borderline range, illustrates the clinical relevance of a borderline QFT in this setting. Infliximab is a monoclonal antibody directed against TNF- $\alpha$. TNF- $\alpha$ plays a major role in recruitment and organisation of mononuclear cells into well-structured granulomas. Anti-TNF therapy can result in disintegration of granulomas and reactivation TB [4]. However, this cannot be extrapolated directly to biologicals with a different mechanism of action and some, such as rituximab, are even considered safe in this regard [5]. Yet, even in the screening era, the risk has remained increased, probably reflecting suboptimal performance of diagnostic tests for LTBI in this setting [6]. In our patient, the positive TST screening result was unfortunately overlooked. Of note, the effect of BCG vaccination, if given before 1 year of age, on the TST is negligible beyond 10 years after vaccination [7]. Thus, the TST should have been qualified as true positive anyway, which was strengthened by the self-reported TB contact. According to the manufacturer's cut-off, her QFT screening result of $0.22 \mathrm{IU} \cdot \mathrm{mL}^{-1}$ was indeed negative. This low value can be explained by the decreased sensitivity of QFT for detection of a remote M. tuberculosis infection [8]. However, recent studies have shown that a result just below the cut-off includes patients with true M. tuberculosis infection [3,9], and additional data support and extend this notion [10]. One retrospective study showed that individuals with a borderline QFT result (defined as 0.20-0.34 IU $\cdot \mathrm{mL}^{-1}$ ) developed active TB significantly more often compared to those with a negative result [11]. However, further research is needed to corroborate our observation, e.g. by retrospective analysis of quantitative QFT results in all patients who developed active TB despite screening. In our opinion, until more data are available, lowering the QFT cut-off should be limited to patients who will receive significant immunosuppression and should not be applied in normal or low-risk settings.

This case emphasises the value of TST irrespective of BCG vaccination and shows that a borderline QFT result in a patient screened before immunosuppression should be considered as a risk factor for reactivation TB. In this setting, a borderline QFT result with or without any other risk factor, be it origin, known exposure, past or present positive TST result and/or suggestive abnormalities on chest radiography, in our opinion, justifies preventive therapy. 
Jonathan W. Uzorka ${ }^{1}$, Nathalie M. Delfos ${ }^{2}$, Anne M.C. Witte ${ }^{3}$, Henk Scheper ${ }^{1}$, Dick van Soolingen ${ }^{4}$ and Sandra M. Arend ${ }^{1}$

${ }^{1}$ Dept of Infectious Diseases, Leiden University Medical Center, Leiden, The Netherlands. ${ }^{2}$ Dept of Internal Medicine, Alrijne Ziekenhuis, Leiderdorp, The Netherlands. ${ }^{3}$ Dept of Gastroenterology and Hepatology, Alrijne Ziekenhuis, Leiderdorp, The Netherlands. ${ }^{4}$ Tuberculosis Reference Laboratory, National Institute for Public Health and the Environment, Bilthoven, The Netherlands.

Correspondence: Jonathan W. Uzorka, Dept of Infectious Diseases, C5-P, Leiden University Medical Center, Albinusdreef 2, 2333 ZA Leiden, The Netherlands. E-mail: j.w.uzorka@lumc.nl

Received: May 072018 | Accepted after revision: June 122018

Conflict of interest: None declared.

\section{References}

1 Migliori GB, Sotgiu G, Rosales-Klintz S, et al. ERS/ECDC Statement: European Union standards for tuberculosis care, 2017 update. Eur Respir J 2018; 51: 1702678.

2 Bumbacea D, Arend SM, Eyuboglu F, et al. The risk of tuberculosis in transplant candidates and recipients: a TBNET consensus statement. Eur Respir J 2012; 40: 990-1013.

3 Uzorka JW, Kroft LJM, Bakker JA, et al. Proof of concept that most borderline QuantiFERON results are true antigen-specific responses. Eur Respir J 2017: 50: 1701630.

4 Solovic I, Sester M, Gomez-Reino JJ, et al. The risk of tuberculosis related to tumour necrosis factor antagonist therapies: a TBNET consensus statement. Eur Respir J 2010; 36: 1185-1206.

5 Cantini F, Nannini C, Niccoli L, et al. Risk of tuberculosis reactivation in patients with rheumatoid arthritis, ankylosing spondylitis, and psoriatic arthritis receiving non-anti-TNF-targeted biologics. Mediators Inflamm 2017; 2017: 8909834

6 Ai JW, Zhang S, Ruan QL, et al. The risk of tuberculosis in patients with rheumatoid arthritis treated with tumor necrosis factor-alpha antagonist: a metaanalysis of both randomized controlled trials and registry/cohort studies. J Rheumatol 2015; 42: 2229-2237.

7 Farhat M, Greenaway C, Pai M, et al. False-positive tuberculin skin tests: what is the absolute effect of BCG and non-tuberculous mycobacteria? Int J Tuberc Lung Dis 2006; 10: 1192-1204.

8 Leyten EM, Arend SM, Prins C, et al. Discrepancy between Mycobacterium tuberculosis-specific gamma interferon release assays using short and prolonged in vitro incubation. Clin Vaccine Immunol 2007; 14: 880-885.

9 Nemes E, Rozot V, Geldenhuys $\mathrm{H}$, et al. Optimization and interpretation of serial QuantiFERON testing to measure acquisition of Mycobacterium tuberculosis infection. Am J Respir Crit Care Med 2017; 196: 638-648.

10 Uzorka JW, Bossink AWJ, Franken WPJ. Borderline QuantiFERON results and the distinction between specific responses and test variability. Tuberculosis 2018; 111: 102-108.

11 Jonsson J, Westman A, Bruchfeld J, et al. A borderline range for QuantiFERON Gold In-Tube results. PLoS One 2017; 12: e0187313. 